\title{
ON EXTREMAL EXTENSIONS OF REGULAR CONTENTS AND MEASURES
}

\author{
WOLFGANG ADAMSKI \\ (Communicated by Andrew Bruckner)
}

\begin{abstract}
Within the framework of regular extensions of contents and measures we show that every regular content admits an extremal extension. Under an additional assumption we can also prove the existence of extremal measure extensions.
\end{abstract}

\section{INTRODUCTION}

It is well known that there are a lot of different characterizations of extremal extensions of contents and measures (see $[11,6,7,4,9])$. As for existence results, however, only one general existence theorem for extremal extensions of contents, due to Plachky [11], is known, whereas extremal measure extensions are considered always for special situations (e.g., if the target $\sigma$-algebra is generated from a given $\sigma$-algebra by adjunction of a family of pairwise disjoint sets $[6,4])$, since, in general, extremal measure extensions do not exist (see [11, $13])$.

In this note we consider two lattices $\mathscr{K}, \mathscr{L}$ of subsets of some set $X$ with $\mathscr{K} \subset \mathscr{L}$, and we show that every $\mathscr{K}$-regular content defined on the algebra generated by $\mathscr{K}$ has an extremal extension to an $\mathscr{L}$-regular content on the algebra generated by $\mathscr{L}$. This result, a generalization of Plachky's existence theorem, is used to prove that, under an additional assumption, every $\mathscr{K}$-regular measure has an extremal extension to an $\mathscr{L}$-regular measure. Various applications of this latter result are given.

Now we fix the notation. $X$ will always denote an arbitrary set. A paving (in $X)$ is a subset of $\mathscr{P}(X)$, the power set of $X$, which contains the empty set. A paving that is closed under finite (countable) intersections and finite unions is called a lattice $(\delta$-lattice).

If $\mathscr{K} \subset \mathscr{P}(X)$ is a lattice, then $\mathscr{F}(\mathscr{K}):=\{F \subset X: F \cap K \in \mathscr{K}$ for all $K \in$ $\mathscr{K}\}$ denotes the lattice of the so-called "local $\mathscr{K}$-sets". Obviously, $X \in \mathscr{F}(\mathscr{K})$ and $\mathscr{K} \subset \mathscr{F}(\mathscr{K})$; in addition, we have $\mathscr{K}=\mathscr{F}(\mathscr{K})$ iff $X \in \mathscr{K}$.

Let $\mathscr{C}$ be a paving in $X$. Then $\alpha(\mathscr{C}), \sigma(\mathscr{C})$ denote the algebra, $\sigma$-algebra generated by $\mathscr{C}$, respectively. $\mathscr{C}$ is said to be semicompact if every countable

Received by the editors November 9, 1992.

1991 Mathematics Subject Classification. Primary 28A12, 28C15. 
subfamily of $\mathscr{C}$ having the finite intersection property has a nonvoid intersection.

If $\mathscr{K}, \mathscr{L}$ are pavings in $X$, then $\mathscr{K}$ is said to be sequentially dominated by $\mathscr{L}$ if, whenever $\left(K_{n} \in \mathscr{K}\right)_{n \in \mathbb{N}}$ and $K_{n} \downarrow \phi$, there exists a sequence $\left(L_{n} \in\right.$ $\mathscr{L})_{n \in \mathbb{N}}$ such that $L_{n} \downarrow \phi$ and $K_{n} \subset L_{n}$ for all $n \in \mathbb{N}$. Note that a semicompact paving is sequentially dominated by any paving $\mathscr{L}$ with $X \in \mathscr{L}$.

By a content (measure) we always understand a $[0, \infty)$-valued, finitely (countably) additive set function defined on an algebra ( $\sigma$-algebra).

Let $\mathscr{A} \subset \mathscr{P}(X)$ be an algebra and $\lambda$ a content on $\mathscr{A}$. With $\lambda$ there are associated two set functions $\lambda^{*}$ and $\lambda_{*}$ defined, for all $E \subset X$, by $\lambda^{*}(E):=$ $\inf \{\lambda(A): E \subset A \in \mathscr{A}\}$ and $\lambda_{*}(E):=\sup \{\lambda(A): E \supset A \in \mathscr{A}\}$. If $\mathscr{C} \subset \mathscr{A}$ is a paving, then $\lambda$ is said to be $\mathscr{C}$-regular if $\lambda(A)=\sup \{\lambda(C): C \in \mathscr{C}, C \subset A\}$ holds for all $A \in \mathscr{A}$.

Let $X$ be a topological space. Any set of the form $\{f=0\}$, where $f$ is a real-valued continuous function on $X$, is called a zero-set in $X$. We denote by $\mathscr{Z}(X), \mathscr{F}(X), \mathscr{K}(X)$, and $\mathscr{K}_{0}(X)$ the family of all zero-, closed, compact, and compact $G_{\delta}$-sets in $X$, respectively. Note that $X$ is countably compact (pseudocompact) iff $\mathscr{F}(X)(\mathscr{Z}(X))$ is semicompact. $\mathscr{B}_{0}(X):=\sigma(\mathscr{Z}(X))$ $(\mathscr{B}(X):=\sigma(\mathscr{F}(X)))$ denotes the Baire (Borel) $\sigma$-algebra in $X$. Any measure defined on $\mathscr{B}_{0}(X)(\mathscr{B}(X))$ is called a Baire (Borel) measure on $X . \mathscr{F}(X)$ regular Borel measures are simply called regular. On the other hand, a $\mathscr{K}(X)$ regular Borel measure on a Hausdorff space $X$ is called a Radon measure. According to [12, Theorem 18, Part I] we have

\subsection{Lemma. Every Baire measure on a topological space $X$ is $\mathscr{Z}(X)$-regular.}

Finally, for any convex subset $D$ of some real vector space, we write ex $D$ for the set of extreme points of $D$.

\section{THE MAIN RESULTS}

Consider a content $\lambda$ defined on an algebra $\mathscr{A} \subset \mathscr{P}(X)$, and let $\mathscr{L} \subset \mathscr{P}(X)$ be a lattice such that $\mathscr{A} \subset \alpha(\mathscr{L})$. We denote by ba $(\lambda, \mathscr{L})$ the convex set of all $\mathscr{L}$-regular contents on $\alpha(\mathscr{L})$ that extend $\lambda$. Observe that, for the case of an algebra $\mathscr{L}$, ba $(\lambda, \mathscr{L})$ is the family of all contents on $\mathscr{L}$ which are extensions of $\lambda$.

2.1. Lemma. For $\mu \in \mathrm{ba}(\lambda, \mathscr{L})$, we have

$$
\mu \in \operatorname{ex} \mathrm{ba}(\lambda, \mathscr{L}) \text { iff } \mu \in \operatorname{ex} \mathrm{ba}(\lambda, \alpha(\mathscr{L})) \text {. }
$$

Proof. Let $\mu \in \operatorname{exba}(\lambda, \mathscr{L})$, and assume $\mu=2^{-1}\left(\mu_{1}+\mu_{2}\right)$ with $\mu_{1}, \mu_{2} \in$ $\mathrm{ba}(\lambda, \alpha(\mathscr{L}))$. As $2^{-1} \mu_{i} \leq \mu$ for $i=1,2$ and $\mu$ is $\mathscr{L}$-regular, we infer $\mu_{1}, \mu_{2} \in \mathrm{ba}(\lambda, \mathscr{L})$, so $\mu_{1}=\mu_{2}$. Thus $\mu \in \operatorname{exba}(\lambda, \alpha(\mathscr{L}))$. The other part of the claim is obvious.

2.2. Lemma. Let $\mathscr{K} \subset \mathscr{P}(X)$ be a lattice with $X \in \mathscr{K}$, and let $\lambda$ be a $\mathscr{K}$ regular content on $\alpha(\mathscr{K})$. For a given $Q \in \mathscr{P}(X) \backslash \mathscr{K}$, denote by $\check{\mathscr{K}}$ the lattice generated by $\mathscr{K} \cup\{Q\}$ and define $\check{\mu}(D):=\sup \left\{\lambda_{*}(S \backslash Q)+\lambda^{*}(S \cap Q): S \in \check{\mathscr{K}}, S \subset\right.$ $D\}$ for $D \in \alpha(\check{\mathscr{K}})$. Then $\check{\mu} \in \operatorname{ex} \mathrm{ba}(\lambda, \check{\mathscr{K}})$.

Proof. By [1, Lemma 2.1], $\check{\mu} \in \mathrm{ba}(\lambda, \breve{\mathscr{K}})$. Thus, in view of 2.1 and [11, Theorem 1 and the associated Remark 2], it suffices to show that for any $\varepsilon>0$ 
there is an $\alpha(\mathscr{K})$-set $L$ satisfying $\check{\mu}(L \Delta Q)<\varepsilon$. For this purpose choose $L \in \alpha(\mathscr{K})$ such that $Q \subset L$ and $\lambda(L)<\lambda^{*}(Q)+\varepsilon$. Since $X \in \mathscr{K}$, we have $\check{\mathscr{K}}=\left\{(Q \cap K) \cup K^{\prime}: K, K^{\prime} \in \mathscr{K}\right\}$. Consequently, for every $S \in \check{\mathscr{K}}$ with $S \subset L \Delta Q=L \backslash Q$ we have $S \in \mathscr{K}$, and hence $\lambda_{*}(S \backslash Q)+\lambda^{*}(S \cap Q)=\lambda_{*}(S \backslash Q) \leq$ $\lambda(S) \leq \lambda_{*}(L \backslash Q)=\lambda(L)-\lambda^{*}(Q)<\varepsilon$, which proves our claim.

Now we consider two lattices $\mathscr{K}, \mathscr{L}$ of subsets of $X$ satisfying $\mathscr{K} \subset \mathscr{L}$ together with a $\mathscr{K}$-regular content $\lambda$ on $\alpha(\mathscr{K})$. It is well known that ba $(\lambda, \mathscr{L})$ is nonvoid (see [1, Theorem 3.2] or [3, Theorem 2.1]). The following theorem, the central result of this note, states that the convex set $\mathrm{ba}(\lambda, \mathscr{L})$ even has extreme points. Its proof is based on a suitable modification of the proof of [1, Theorem 2.2].

2.3. Theorem. Let $\mathscr{K}, \mathscr{L}$ be lattices of subsets of $X$ with $\mathscr{K} \subset \mathscr{L}$. Then ex ba $(\lambda, \mathscr{L}) \neq \varnothing$ for every $\mathscr{K}$-regular content $\lambda$ on $\alpha(\mathscr{K})$.

Proof. W.l.o.g. we can assume $X \in \mathscr{K}$. (Otherwise one might introduce the lattices $\mathscr{K}^{\prime}=\mathscr{K} \cup\{X\}$ and $\mathscr{L}^{\prime}=\mathscr{L} \cup\{X\}$ since ba $(\lambda, \mathscr{L})=\mathrm{ba}\left(\lambda, \mathscr{L}^{\prime}\right)$.) We consider ordered pairs $(\mathscr{M}, \mu)$ where $\mathscr{M}$ is a lattice of subsets of $X$ and $\mu$ is a $\mathscr{M}$-regular content on $\alpha(\mathscr{M})$. Partially order these pairs in the following way: $(\mathscr{M}, \mu) \leq\left(\mathscr{M}^{\prime}, \mu^{\prime}\right)$ iff $\mathscr{M} \subset \mathscr{M}^{\prime}$ and $\mu^{\prime}$ is an extension of $\mu$.

Define $\Gamma:=\{(\mathscr{M}, \mu): \mathscr{K} \subset \mathscr{M} \subset \mathscr{L}$ and $\mu \in$ ex ba $(\lambda, \mathscr{M})\}$. Note that $(\mathscr{K}, \lambda) \in \Gamma$.

(1) $\Gamma$ is inductively ordered.

Let $\left(\mathscr{M}_{i}, \mu_{i}\right)_{i \in I}$ be a chain in $\Gamma$. Then $\mathscr{M}:=\bigcup_{i} \mathscr{M}_{i}$ is a lattice which satisfies $\mathscr{K} \subset \mathscr{M} \subset \mathscr{L}$ and $\alpha(\mathscr{M})=\bigcup_{i} \alpha\left(\mathscr{M}_{i}\right)$. For $A \in \alpha(\mathscr{M})$ define $\mu(A):=\mu_{i}(A)$ provided that $A \in \alpha\left(\mathscr{M}_{i}\right)$. Then $\mu$ is a $\mathscr{M}$-regular content on $\alpha(\mathscr{M})$ that extends every $\mu_{i}$. Thus $\left(\mathscr{M}_{i}, \mu_{i}\right) \leq(\mathscr{M}, \mu)$ for any $i \in I$. In particular, $\mu$ is an extension of $\lambda$, i.e., $\mu \in \mathrm{ba}(\lambda, \mathscr{M})$. To prove $\mu \in \operatorname{exba}(\lambda, \mathscr{M})$, consider $\rho_{1}, \rho_{2} \in \mathrm{ba}(\lambda, \mathscr{M})$ with $\mu=2^{-1}\left(\rho_{1}+\rho_{2}\right)$. Fix some $i_{0} \in I$, and set $\hat{\rho}_{j}:=\rho_{j} \mid \alpha\left(\mathscr{M}_{i_{0}}\right)$ for $j=1,2$. Then $\hat{\rho}_{1}, \hat{\rho}_{2} \in \operatorname{ba}\left(\lambda, \alpha\left(\mathscr{M}_{i_{0}}\right)\right)$ and $\mu_{i_{0}}=$ $2^{-1}\left(\hat{\rho}_{1}+\hat{\rho}_{2}\right)$. Since $\mu_{i_{0}} \in \operatorname{exba}\left(\lambda, \mathscr{M}_{i_{0}}\right)$, we infer $\hat{\rho}_{1}=\hat{\rho}_{2}$ from 2.1. Now consider an arbitrary $A \in \alpha(\mathscr{M})$. Then $A \in \alpha\left(\mathscr{M}_{i_{0}}\right)$ for some $i_{0} \in I$, so $\rho_{1}(A)=\hat{\rho}_{1}(A)=\hat{\rho}_{2}(A)=\rho_{2}(A)$. Thus $\rho_{1}=\rho_{2}$, and hence $\mu \in \operatorname{exba}(\lambda, \mathscr{M})$, which implies $(\mathscr{M}, \mu) \in \Gamma$. So (1) holds true.

(2) By Zorn's lemma, there is a maximal element $(\widetilde{\mathscr{M}}, \rho)$ in $\Gamma$. We will show that $\widetilde{\mathscr{M}}=\mathscr{L}$, which implies that $\rho$ is the desired element of ex ba $(\lambda, \mathscr{L})$.

Assume there is a set $Q \in \mathscr{L} \backslash \widetilde{\mathscr{M}}$. Denoting by $\check{\mathscr{K}}$ the lattice generated by $\widetilde{\mathscr{M}} \cup\{Q\}$ there exists, by 2.2 , an element $\check{\mu} \in \operatorname{exba}(\rho, \check{\mathscr{K}})$. Next we shall prove $\check{\mu} \in \operatorname{ex}$ ba $(\lambda, \check{\mathscr{K}})$, which implies $(\check{\mathscr{K}}, \check{\mu}) \in \Gamma$. On the other hand, we have $(\widetilde{\mathscr{M}}, \rho) \leq(\check{\mathscr{K}}, \check{\mu})$ and $\widetilde{\mathscr{M}} \neq \check{\mathscr{K}}$, which, however, contradicts the maximality of $(\widetilde{\mathscr{M}}, \rho)$.

It is obvious that $\check{\mu} \in \mathrm{ba}(\lambda, \check{\mathscr{K}})$. To prove the extremality of $\check{\mu}$, let $\check{\mu}$ $=2^{-1}\left(\mu_{1}+\mu_{2}\right)$ with $\mu_{1}, \mu_{2} \in \mathrm{ba}(\lambda, \check{\mathscr{K}})$. Define $\tilde{\mu}_{i}:=\mu_{i} \mid \alpha(\widetilde{\mathscr{M}}), i=1,2$. For $A \in \alpha(\widetilde{\mathscr{M}})$ we have $\rho(A)=\check{\mu}(A)=2^{-1}\left(\tilde{\mu}_{1}(A)+\tilde{\mu}_{2}(A)\right)$, i.e., $\rho=$ $2^{-1}\left(\tilde{\mu}_{1}+\tilde{\mu}_{2}\right)$. Since $\tilde{\mu}_{i} \in \mathrm{ba}(\lambda, \alpha(\widetilde{\mathscr{M}}))$ for $i=1,2$ and $\rho \in \operatorname{exba}(\lambda, \widetilde{\mathscr{M}})$, we infer $\tilde{\mu}_{1}=\tilde{\mu}_{2}=\rho$ from 2.1. Thus $\mu_{1}, \mu_{2} \in \mathrm{ba}(\rho, \alpha(\check{\mathscr{K}}))$. As $\check{\mu}$ is an element of ex $\mathrm{ba}(\rho, \check{\mathscr{K}})$, we have $\mu_{1}=\mu_{2}$ by 2.1 . This proves $\check{\mu} \in \operatorname{ex} \mathrm{ba}(\lambda, \check{\mathscr{K}})$. 
Remark. If $\mathscr{K}$ and $\mathscr{L}$ are algebras in $X$ with $\mathscr{K} \subset \mathscr{L}$, and if $\lambda$ is a content on $\mathscr{K}$, then ba $(\lambda, \mathscr{L})$ is the family of all contents on $\mathscr{L}$ that extend $\lambda$. For this special case Plachky [11] proved the existence of extreme points by a KreinMilman argument. However, in our more general situation we cannot use this method since $\mathrm{ba}(\lambda, \mathscr{L})$ is, in general, not closed in $\mathrm{ba}(\lambda, \alpha(\mathscr{L}))$ with respect to the topology of setwise convergence.

Next we are concerned with extremal extensions of regular measures. For this purpose we consider two $\sigma$-algebras $\mathscr{A}, \mathscr{B}$ in $X$ satisfying $\mathscr{K} \subset \mathscr{A} \subset$ $\sigma(\mathscr{F}(\mathscr{K})), \mathscr{L} \subset \mathscr{B} \subset \sigma(\mathscr{F}(\mathscr{L}))$, and $\mathscr{A} \subset \mathscr{B}$. (Note that $X \in \mathscr{K} \quad(X \in \mathscr{L})$ implies $\mathscr{A}=\sigma(\mathscr{K})(\mathscr{B}=\sigma(\mathscr{L}))$. Furthermore, for any $\mathscr{K}$-regular measure $\lambda$ on $\mathscr{A}$ we write $\mathrm{ca}(\lambda, \mathscr{L}, \mathscr{B})$ for the convex set of all $\mathscr{L}$-regular measures on $\mathscr{B}$ that extend $\lambda$. It is shown in $[11,13]$ that the implication $\mathrm{ca}(\lambda, \mathscr{L}, \mathscr{B}) \neq$ $\phi \Rightarrow \operatorname{exca}(\lambda, \mathscr{L}, \mathscr{B}) \neq \phi$ is, in general, not true, even if $\mathscr{K}$ and $\mathscr{L}$ are (countably generated [13]) $\sigma$-algebras (and hence $\mathscr{K}=\mathscr{A}, \mathscr{L}=\mathscr{B}$ ).

However, under an additional assumption, we have

2.4. Theorem. Let $\mathscr{L}$ be a $\delta$-lattice that is sequentially dominated by $\sigma(\mathscr{F}(\mathscr{K}))$. Then ex $\mathrm{ca}(\lambda, \mathscr{L}, \mathscr{B}) \neq \phi$ for every $\mathscr{K}$-regular measure $\lambda$ on $\mathscr{A}$.

Proof. By 2.3 , there exists $\tilde{\rho} \in \operatorname{ex} \operatorname{ba}(\tilde{\lambda}, \mathscr{L})$ where $\tilde{\lambda}:=\lambda \mid \alpha(\mathscr{K})$. Setting $\rho:=\tilde{\rho} \mid \mathscr{L}$, we infer from the proof of $[1$, Theorem 3.3(a) $]$ that the set function $\mu$ defined by $\mu(B):=\sup \{\rho(L): L \in \mathscr{L}, L \subset B\}, B \in \mathscr{B}$, is an element of $\mathrm{ca}(\lambda, \mathscr{L}, \mathscr{B})$. To prove the extremality of $\mu$, consider $\mu_{1}, \mu_{2} \in \mathrm{ca}(\lambda, \mathscr{L}, \mathscr{B})$ such that $\mu=2^{-1}\left(\mu_{1}+\mu_{2}\right)$ holds. Then $\tilde{\mu}_{i}:=\mu_{i} \mid \alpha(\mathscr{L}) \in \mathrm{ba}(\tilde{\lambda}, \mathscr{L})$ for $i=1,2$ and $\tilde{\rho}=2^{-1}\left(\tilde{\mu}_{1}+\tilde{\mu}_{2}\right)$. Since $\tilde{\rho} \in \operatorname{ex}$ ba $(\tilde{\lambda}, \mathscr{L})$, we have $\tilde{\mu}_{1}=\tilde{\mu}_{2}$ and, hence, $\mu_{1}=\mu_{2}$ by $\mathscr{L}$-regularity. Thus $\mu \in \operatorname{ex} \mathrm{ca}(\lambda, \mathscr{L}, \mathscr{B})$.

\section{Applications}

In this section we will give several applications of Theorem 2.4. The first one is concerned with extremal extensions of Baire to Borel measures. Recall from [1] that a topological space $X$ is said to be Baire-dominated if $\mathscr{F}(X)$ is sequentially dominated by $\mathscr{B}_{0}(X)$. For this class of topological spaces we have the following result.

3.1. Theorem. Let $X$ be a Baire-dominated topological space. Then every Baire measure on $X$ admits an extremal extension to a regular Borel measure on $X$. Proof. In view of 1.1, our claim follows with $\mathscr{K}=\mathscr{Z}(X)$ and $\mathscr{L}=\mathscr{F}(X)$ from 2.4.

Note that a topological space $X$ is Baire-dominated if $X$ is a cb-space [1] or $X$ is cozero-dominated [10]. In particular, $X$ is Baire-dominated if $X$ is countably compact or $X$ is normal and countably paracompact.

3.2. Theorem. Let $X$ be a Hausdorff space. Then every $\mathscr{K}_{0}(X)$-regular measure on $\sigma\left(\mathscr{K}_{0}(X)\right)$ has an extremal extension to a Radon measure on $X$.

Proof. Set $\mathscr{K}=\mathscr{K}_{0}(X), \mathscr{L}=\mathscr{K}(X), \mathscr{A}=\sigma(\mathscr{K})$, and $\mathscr{B}=\mathscr{B}(X)$. Then $\mathscr{F}(X) \subset \mathscr{F}(\mathscr{L})$ and hence $\mathscr{B} \subset \sigma(\mathscr{F}(\mathscr{L}))$. Thus our claim follows from 2.4.

3.3. Theorem. Let $\tau_{1}, \tau_{2}$ be topologies on a set $X$ where $\tau_{2}$ is finer than $\tau_{1}$. For $i=1,2$ let $\mathscr{F}_{i}\left(\mathscr{Z}_{i}\right)$ be the family of closed (zero-) sets in $X$ pertaining to the topology $\tau_{i}$. 
(a) Assume that $\mathscr{F}_{2}$ is sequentially dominated by $\sigma\left(\mathscr{F}_{1}\right)$. Then every $\mathscr{F}_{1}$ regular Borel measure on $\left(X, \tau_{1}\right)$ has an extremal extension to a $\mathscr{F}_{2}$-regular Borel measure on $\left(X, \tau_{2}\right)$.

(b) Assume that $\mathscr{Z}_{2}$ is sequentially dominated by $\sigma\left(\mathscr{Z}_{1}\right)$. Then every Baire measure on $\left(X, \tau_{1}\right)$ has an extremal extension to a Baire measure on $\left(X, \tau_{2}\right)$. Proof. (a) follows with $\mathscr{K}=\mathscr{F}$ and $\mathscr{L}=\mathscr{F}_{2}$ from 2.4. In view of 1.1, (b) follows with $\mathscr{K}=\mathscr{Z}_{1}$ and $\mathscr{L}=\mathscr{Z}_{2}$ from 2.4.

The domination property in $3.3(\mathrm{a})(3.3(\mathrm{~b}))$ is, in particular, satisfied if $\left(X, \tau_{2}\right)$ is countably compact (pseudocompact). Thus $3.3(\mathrm{a})$ is a common generalization of [8, Theorem $9 ; 2$, Corollary $3.2 ; 3$, Corollary 2.3$]$, whereas $3.3(\mathrm{~b})$ generalizes [2, Corollary 3.4].

Our next result is concerned with the existence of extremal preimage measures.

3.4. Theorem. Let $X, Y$ be arbitrary sets, and let $f$ be a mapping from $X$ onto $Y$. Furthermore, let $\mathscr{D} \subset \mathscr{P}(Y)$ be a lattice and $\mathscr{C} \subset \mathscr{P}(X)$ a $\delta$-lattice such that $f^{-1}(\mathscr{D}) \subset \mathscr{C}$ holds. If $\mathscr{C}$ is sequentially dominated by $\sigma\left(f^{-1}(\mathscr{D})\right)$, then for any $\mathscr{D}$-regular measure $\nu$ on $\sigma(\mathscr{D})$, the convex set of $\mathscr{C}$-regular measures $\mu$ on $\sigma(\mathscr{C})$ with $\nu=f(\mu)$ (i.e., $\nu(B)=\mu\left(f^{-1}(B)\right.$ ) for all $B \in \sigma(\mathscr{D})$ ) has an extreme point.

Proof. Put $\gamma\left(f^{-1}(B)\right):=\nu(B)$ for $B \in \sigma(\mathscr{D})$. Then $\gamma$ is a $f^{-1}(\mathscr{D})$-regular measure on $f^{-1}(\sigma(\mathscr{D}))=\sigma\left(f^{-1}(\mathscr{D})\right)$. By $2.4, \gamma$ has an extremal extension to a $\mathscr{C}$-regular measure $\check{\mu}$ on $\sigma(\mathscr{C})$. It is easy to see that $\check{\mu}$ is an extreme point of the set of all $\mathscr{C}$-regular measures $\mu$ on $\sigma(\mathscr{C})$ with $\nu=f(\mu)$.

3.5. Corollary. Let $X, Y$ be topological spaces, and let $f$ be a continuous mapping from $X$ onto $Y$.

(a) If $\mathscr{Z}(X)$ is sequentially dominated by $f^{-1}\left(\mathscr{B}_{0}(Y)\right)$, then, for any Baire measure $\nu$ on $Y$, the convex set of Baire measures $\mu$ on $X$ with $\nu=f(\mu)$ has an extreme point.

(b) If $\mathscr{F}(X)$ is sequentially dominated by $f^{-1}(\mathscr{B}(Y))$, then, for any regular Borel measure $\nu$ on $Y$, the convex set of regular Borel measures $\mu$ on $X$ with $\nu=f(\mu)$ has an extreme point.

Remarks. (a) Note that the domination condition in 3.5(a) (3.5(b)) is satisfied if $X$ (and hence also $Y$ ) is pseudocompact (countably compact). Thus 3.5(b) generalizes [8, Theorem 10].

(b) Various characterizations of extremal preimage measures can be found in $[5, \S 3]$.

\section{REFERENCES}

1. W. Adamski, Extensions of tight set functions with applications in topological measure theory, Trans. Amer. Math. Soc. 283 (1984), 353-368.

2. G. Bachman and A. Sultan, Extensions of regular lattice measures with topological applications, J. Math. Anal. Appl. 57 (1977), 539-559.

3. __ On regular extensions of measures, Pacific J. Math. 86 (1980), 389-395.

4. D. Bierlein and W. J. A. Stich, On the extremality of measure extensions, Manuscripta Math. 63 (1989), 89-97.

5. S. Graf, Induced $\sigma$-homomorphisms and a parametrization of measurable sections via extremal preimage measures, Math. Ann. 247 (1980), 67-80. 
6. W. Hackenbroch, Measures admitting extremal extensions, Arch. Math. 49 (1987), 257-266.

7. —_ Measure extensions by conditional atoms, Math. Z. 200 (1989), 347-352.

8. J. Hardy and H. E. Lacey, Extensions of regular Borel measures, Pacific J. Math. 24 (1968), 277-282.

9. Z. Lipecki, On extreme extensions of quasi-measures, Arch. Math. 58 (1992), 288-293.

10. H. Ohta and K.-I. Tamano, Topological spaces whose Baire measure admits a regular Borel extension, Trans. Amer. Math. Soc. 317 (1990), 393-415.

11. D. Plachky, Extremal and monogenic additive set functions, Proc. Amer. Math. Soc. 54 (1976), 193-196.

12. V. S. Varadarajan, Measures on topological spaces, Amer. Math. Soc. Transl. Ser. 2, vol. 48, Amer. Math. Soc., Providence, RI, 1965, pp. 161-228.

13. H. von Weizsäcker, Remark on extremal measure extensions, Lecture Notes in Math., vol. 794, Springer-Verlag, Berlin and New York, 1980, pp. 79-80.

Mathematisches Institut, Universität MÜnChen, Theresienstr. 39, D-80333 MÜNChen, GERMANY 